\title{
Silica Chloride Catalyzed Efficient Synthesis of 2,3-Dihydroquinazoline-4(1h)-Ones Derivatives In Water
}

\author{
Suvarna Lavale and Milind Ubale* \\ Post graduate department of Chemistry, Vasantrao Naik College, India
}

Received: November 07, 2017; Published: November 29, 2017

*Corresponding author: Milind Ubale, Post graduate department of Chemistry, Vasantrao Naik College, Aurangabad, India; Email: drmilindubale@gmail.com

\begin{abstract}
Describe method consist of eco-friendly procedure for the preparation of 2,3-dihydroquinazoline-4(1H)-ones from equimolar 2 -aminobenzamide and substituted aromatic aldehydes in presence of $20 \mathrm{~mol} \%$ of silica chloride. Green impact of reaction significantly enhanced due to use of water as solvent and recyclable silica-chloride as catalyst. Good to excellent yield of products, simple working strategy and easy purification are the advantage of present methodology
\end{abstract}

Keywords: Quinazoline; Silica-chloride catalyst; Water mediated; Green methodology

\section{Introduction}

Search of expeditious and cost-effective methodologies to replace tedious, low productive traditional methodologies gains its own importance. Now a day's green methodologies has attract significant attention and environmentally benign, recyclable, chap solid catalysts get ultimate reputation. Such methodology offers to obtained complex pharmaceutically important molecules or intermediate by possibly viable ways. Such methodologies shine with imminent light when water incorporates as solvent, due to its non-toxic, green, cheap nature and biochemical consequence $[1,2]$.

Quinazoline has been occupied distinct position in nitrogen containing heterocycles due to its spectacular wide spectrum of pharmaceutical properties. Various reports of quinazoline underline its widely biopharmaceutical activity like, anticancer [35], antibacterial [6-8], antiinflammation [9,10], antituberculosis [11], antihypertension [12] and antidiabeties [13]. Such wide spectrum of quinazoline strongly demands possible derivatisation to test out for further pharmaceutical possibilities. Various methods have been proposed to obtained quinazoline analogues using catalysts like ammonium bromide[14], Zirconyl chloride [15] Heteropoly acids [16], Gallium (III) triflate [17], Titanium oxide nano-particles [18], Starch solution [19], cyanuric chloride [20] and Cyclodextrin sulphonic acid [21].Most of these methodologies are suffers from long reaction time, high temperature, use of expensive catalyst and tedious work procedures. 'On water' reports [22] of quinazoline synthesis by using expensive catalyst increase cost of reaction.
Readily accessible solid support catalyst like Silica chloride for the synthesis one pot quinazoline has not attempted. Such solid supported catalyst simplifies the reaction procedure and do not pass on unpleasant toxic residue to environment. Silica, perhaps most easily available substance and extensively used as support in reactions [23]. Much organic reactions and used silica chloride as efficient catalyst [24-28]. In continuation of our previous research work [29] to develop fast, naturally benign, productive methodology for small and fused heterocyclic compounds, we intended to developed facile, efficient, cost-effective and easy workup method for the synthesis of quinazoline derivatives. Here, introduce facile methodology as shown in scheme 1 for syntheses of quinazoline derivatives (Figure 1).

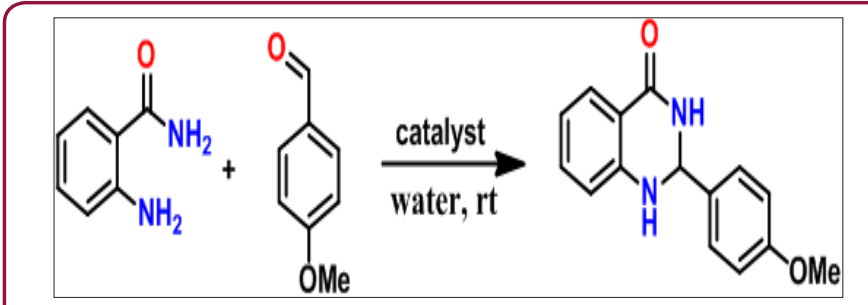

Figure 1: Syntheses of Quinazoline Derivatives.

\section{Experimental}

The reagents and solvents were purchased from Alfa aesar and Aldrich Chemical companies and used without further purification. All compounds obtained were describe for open head capillary tube for their melting point and are uncorrected. The samples 
were analyzed by FT-IR spectroscopy (JASCO FT/IR-460 plus spectrometer). ${ }^{1} \mathrm{HNMR}$ and ${ }^{13} \mathrm{CNMR}$ spectra of compounds were recorded on a Bruker DRX-400 Avance instrument in DMSO-d6.

\section{Preparation of Silica-chloride}

To a well-stirred silica gel (20 g) in DCM $(50 \mathrm{~mL})$ was added to this slow drop wise $\mathrm{SOCl}_{2}(20 \mathrm{~g})$ introduced at room temperature. After stirring for 1-2 hour, the solvent was removed under reduced pressure to dryness. The silica chloride thus obtained was used in the describe experiments as catalyst [28].

\section{General Procedure}

In a RBF containing $20 \mathrm{ml}$ of water was added 2 -aminobezamide $(0.01 \mathrm{~mol} 1.36 \mathrm{gm})$, substituted aldehyde $(0.01 \mathrm{~mol})$ and portion wise $\mathrm{SiO}_{2}-\mathrm{Cl}$ (2 gm 20 mol\%) with stirring. The reaction mixture was stir at room temperature. Progress of reaction was monitor by thin layer chromatography (TLC) using Ethyl acetate-Hexane. After completion of reaction, reaction mixture was filter off and filtrate neutralized by saturated solution of sodium bicarbonate, brine and extracted with ethyl acetate. Organic layer dried on anhydrous sodium sulphate and evaporated in reduced pressure to afford pure product after recrystallization from ethanol. Representative compounds were scan for spectral data and found satisfactory agreement with reported.

\section{Silica Chloride as Reusable Catalyst}

Catalyst obtained after filter off from first reaction cycle, and dried at $110^{\circ} \mathrm{C}$ for 2 hours and used for second cycles. Process was repeated until trace product formation takes place during the course of reaction (Table 2).

\section{Spectral data of representative compounds}

A. 2-phenyl-2,3-dihydroquinazolin-4(1H)-one; (1) m.p.= $219^{\circ} \mathrm{C},{ }^{1} \mathrm{HNMR}$ (400 MHz, DMSO-d6): $\delta=8.27(\mathrm{~s}, 1 \mathrm{H}), 7.61(\mathrm{~d}$, $1 \mathrm{H}), 7.50(\mathrm{~d}, 2 \mathrm{H}), 7.31-7.41(\mathrm{~m}, 3 \mathrm{H}), 7.22(\mathrm{t}, 1 \mathrm{H}), 7.06(\mathrm{~s}, 1 \mathrm{H})$, $6.72(\mathrm{~d}, 1 \mathrm{H}), 6.69(\mathrm{t}, 1 \mathrm{H}), 5.75(\mathrm{~s}, 1 \mathrm{H}) \mathrm{ppm}$; IR (KBr): 3310, $3014,1671,1630,1523 \mathrm{~cm}^{-1}$.

B. 2-(4-methoxyphenyl)-2,3-dihydroquinazolin-4(1H)-one; (2) m.p. $184^{\circ} \mathrm{C},{ }^{1} \mathrm{HNMR}(400 \mathrm{MHz}$, DMSO-d6,) $\delta: 8.28$ (s, 1H), $7.70(\mathrm{~d}, 1 \mathrm{H}), 7.51(\mathrm{~d}, 2 \mathrm{H}), 7.30-7.35(\mathrm{~m}, 1 \mathrm{H}), 7.10(\mathrm{~s}, 1 \mathrm{H}), 7.04$ (d, 2H), $6.83(\mathrm{~d}, \mathrm{~J}=7.6 \mathrm{~Hz}, 1 \mathrm{H}), 6.76(\mathrm{~d}, 1 \mathrm{H}), 5.79(\mathrm{~s}, 1 \mathrm{H}), 3.83(\mathrm{~s}$, 3H); IR (KBr): 3292, 3161, 2830, 1655, 1614, 1543, 1480, 1393, $\mathrm{cm}^{-1}$
C. 2-(p-tolyl)-2,3-dihydroquinazolin-4(1H)-one; (4) m.p.= $224^{\circ} \mathrm{C} .{ }^{1} \mathrm{HNMR}(400 \mathrm{MHz}$, DMSO-d6): $\delta=8.17(\mathrm{~s}, 1 \mathrm{H}), 7.60(\mathrm{~d}$, $1 \mathrm{H}), 7.41(\mathrm{~d}, 2 \mathrm{H}), 7.26-7.17(\mathrm{~m}, 3 \mathrm{H}), 7.02(\mathrm{~s}, 1 \mathrm{H}), 6.75(\mathrm{~d}, 1 \mathrm{H})$, $6.71(\mathrm{t}, 1 \mathrm{H}), 5.70(\mathrm{~s}, 1 \mathrm{H}), 2.31(\mathrm{~s}, 3 \mathrm{H}) \mathrm{ppm}$. IR (KBr): 3312, $3065,1656,1611,1542,1487 \mathrm{~cm}^{-1}$.

D. 2-(4-Bromophenyl)-2,3-dihydroquinazolin-4(1H)- one; (5) m.p. $195^{\circ} \mathrm{C}$; ${ }^{1} \mathrm{HNMR}$ (DMSO-d6, $400 \mathrm{MHz}$ ) $\delta: 8.28$ (s, 1H), 7.58-7.65 (m, 3H), $7.43(\mathrm{~d}, 2 \mathrm{H}), 7.22(\mathrm{~d}, 1 \mathrm{H}), 7.14(\mathrm{~s}, 1 \mathrm{H}), 6.71$ (d, 1H), 6.65 (d, 1H), 5.77 (s, 1H); IR (KBr): 3300, 3174, 3029, $1672,1656,1600,1482,1430.38,1290,1133 \mathrm{~cm}^{-1}$.

\section{Results and Discussions}

Series of reactions were performed to optimized reaction condition including amount of catalyst with respect to yield of product. Room temperature and 'on' water was kept as fix reaction parameters. 2-aminobenzamide and p-methoxy benzaldehyde were taken for model reaction and various reaction condition were applied. At began, model reaction was done without catalyst to ensure necessity of catalyst and was found little productive (Table 1). When silica without surface modification introduces as catalyst (Table 1) reaction was fruitful, but yield of reaction was not satisfactory. Good to excellent results obtained after introduction of silica chloride as catalyst. Amount of silica chloride were vary in search of optimum amount of catalyst from $5 \mathrm{~mol} \%$ to $25 \mathrm{~mol} \%$. It was observed that (Table 1) $20 \mathrm{~mol} \%$ of catalyst offers optimum product.

Silica chloride catalyst reusability study was performed with model reaction (Table 2). First two cycles offers excellent yield of product and productivity decreases after third cycles. It was observed that when reused at fifth time gave $18 \%$ of product, as this reading matches with no-catalyst (Table 1) assumed complete neutralization of efficiency of catalyst and no further reusability were checked. By keeping $20 \mathrm{~mol} \%$ of silica chloride as optimum amount of catalyst further derivatisation were performed. Study of effect of various substituents on the yield of reaction was performed (Table 3). It was observed that electron releasing groups present on aromatic aldehydes offers more conversion of product. $p-\mathrm{OCH}_{3}, p-\mathrm{Br}$, $p$ - $\mathrm{CH}_{3}$ were found highly productive, whereas, $p-\mathrm{NO}_{2}$ benzaldehyde was the less productive substituent. The representative products structures were substantial from their spectral data and found satisfactory agreements with reports in literature [20-30] (Figure 2).

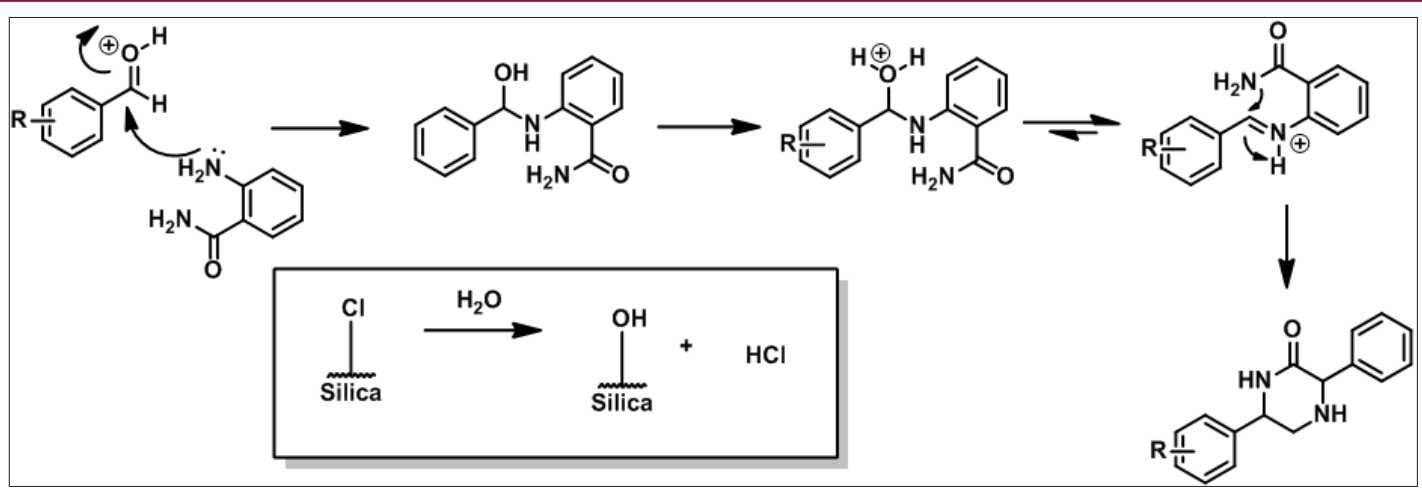

Figure 2: Hypothetical pathway of progress of reaction. 
Table 1: Optimization of amount of Silica-chloride using model reaction strategy.

\begin{tabular}{|c|c|}
\hline SiO2-Cl in $\mathbf{~ m o l \%}$ & Yielda (\%) \\
\hline No Catalyst & 12 \\
\hline 5 & 51 \\
\hline 10 & 58 \\
\hline 15 & 77 \\
\hline 20 & 94 \\
\hline 25 & 95 \\
\hline SiO2(20 mol\%) & 36 \\
\hline
\end{tabular}

Isolated yields Reaction condition: 2-aminobenzamide (0.01 $\mathrm{mol})$, p-methoxy benzaldehyde $(0.01 \mathrm{~mol})$ stirred in water $(20$ $\mathrm{ml})$ at rt. for 60 minutes.
Table 2: Optimization of amount of Silica-chloride using model reaction strategy.

\begin{tabular}{|c|c|c|}
\hline Si02-Cl in mol\% & Time in min. & Yield $^{\text {a }}(\mathbf{\%})$ \\
\hline First lot & 60 & 94 \\
\hline First recycle & 60 & 86 \\
\hline Second recycle & 60 & 78 \\
\hline Third recycle & 120 & 61 \\
\hline Forth recycle & 120 & 55 \\
\hline Fifth recycle & 120 & 18 \\
\hline
\end{tabular}

asolated yields,

Table 3: Showing Quinazoline derivatisation with respect to yield of reaction, time and physical constant of obtained products.

\begin{tabular}{|c|c|c|c|c|c|c|}
\hline Sr. No. & $-\mathbf{R}$ & Compound & Time in min. & Yield ${ }^{\text {a }}$ & M.P. (Lit.) in ${ }^{\circ} \mathrm{C}$ & Ref. \\
\hline 1. & $-\mathrm{H}$ & $3 a$ & 60 & 89 & $219(218-220)$ & [20] \\
\hline 2. & $p$-OMe & $3 \mathrm{~b}$ & 60 & 94 & $184(181-182)$ & [20] \\
\hline 3. & $p-\mathrm{OH}$ & $3 c$ & 60 & 92 & 177 (183-185) & [30] \\
\hline 4. & $p$-Me & $3 \mathrm{~d}$ & 60 & 90 & 224 (227-229) & [20] \\
\hline 5. & $p-\mathrm{Br}$ & $3 e$ & 50 & 95 & $195(197-198)$ & [20] \\
\hline 6. & $p$-Cl & $3 \mathrm{f}$ & 60 & 88 & 208 (206-208) & [20] \\
\hline 7. & $p$-NO2 & $3 g$ & 110 & 73 & $212(214-216)$ & [20] \\
\hline 8. & $p$-N(Me)2 & $3 \mathrm{~h}$ & 50 & 90 & $224(227-229)$ & [20] \\
\hline 9. & $m$-OMe & $3 \mathrm{i}$ & 75 & 90 & 151 (147-149) & [20] \\
\hline 10. & $m-\mathrm{OH}$ & $3 j$ & 60 & 79 & $204(209-210)$ & [30] \\
\hline 11. & $o-\mathrm{Me}$ & $3 \mathrm{k}$ & 50 & 88 & 190 (188-189) & [20] \\
\hline 12. & Furfuraldehyde & 31 & 50 & 91 & $163(166-168)$ & {$[20]$} \\
\hline
\end{tabular}

asolated yields; Reaction condition: 2-aminobenzamide $(0.01 \mathrm{~mol})$, p-methoxy benzaldehyde $(0.01 \mathrm{~mol})$, silica-chloride (20mol\%), stirred in water $(20 \mathrm{ml})$ at $\mathrm{rt}$.

\section{Conclusion}

In conclusion, an efficient and simple method for the synthesis of quinazoline analogues has been described using readily available and cost effective silica chloride as a heterogeneous catalyst. The green reaction profile and mild reaction conditions are main advantage of this method. Reaction takes place at room temperature by simply stirring method, with operational simplicity offers excellent yields.

\section{References}

1. Shaik Karamthulla, Suman Pal, Nasim Khan Lokman H Choudhury (2014) "On-water" synthesis of novel trisubstituted 1,3- thiazoles via microwave-assisted catalyst-free domino reactions 4: 37889-37899.

2. S Narayan, J Muldoon, MG Finn, VV Fokin, HC Kolbe, et al. (2005) "On water": unique reactivity of organic compounds in aqueous suspension. ChemInt 44: 3275-3279.

3. Chandregowda V, Kush AK, Chandrasekara Reddy (2009) G: Synthesis and in vitro antitumor activities of novel 4-anilinoquinazoline derivatives. Eur J Med Chem 44:3046-3055.

4. Al-Rashood ST, Aboldahab IA, Nagi MN, Abouzeid LA, Abdel-Aziz AA, et al. (2006) HI: Synthesis, dihydrofolatereductase inhibition, antitumor testing, and molecular modeling study of some new $4(3 \mathrm{H})$-quinazolinone analogs. Bioorg Med Chem14: 8608-8621.
5. Vasdev N, Dorff PN, Gibbs AR, Nandanan E, Reid LM (2005) HF: Synthesis of 6-acrylamido-4-(2-[18F] fluoroanilino) quinazoline A prospective irreversible EGFR binding probe. J Lablelled Compd Rad 48: 109-115.

6. Rohini R, Muralidhar Reddy P, Shanker K, Hu A, Ravinder (2010) V: Antimicrobial study of newly synthesized 6-substituted indolo[1,2-c] quinazolines. Eur J Med Chem 45: 1200-1205.

7. Antipenko L, Karpenko A, Kovalenko S, Katsev A KomarovskaPorokhnyavets E, et al. (2009) A: Synthesis of new 2-thio$[1,2,4]$ triazolo[1,5-c] quinazoline derivatives and its antimicrobial activity. Chem Pharm Bull 57: 580-585.

8. Jatav V, Kashaw S, Mishra , Vivek Gupta (2008) P: Synthesis and antimicrobial activity of some new 3-[5-(4-substituted)phenyl-1,3,4oxadiazole-2yl]-2-styrylquinazoline-4(3H)-ones. Med Chem Res 17: 205-211.

9. Alagarsamy V, Solomon VR, Dhanabal (2007) K: Synthesis and pharmacological evaluation of some 3-phenyl-2-substituted-3H -quinazolin-4-one as analgesic anti-inflammatory agents. Bioorg Med Chem 15: 235-241.

10. Baba A, Kawamura N, Makino H, Ohta Y, Taketomi S, Sohda T, et al. (1996) $\mathrm{T}$ : Studies on disease-modifying antirheumatic drugs synthesis of novel quinoline quinazoline derivatives and their anti-inflammatory. Effect $1 \mathrm{~J}$ Med Chem 39: 5176-5182.

11. Nandy P, Vishalakshi MT, Bhat (2006) AR: Synthesis and antitubercular activity of Mannich bases of 2-methyl-3H-quinazolin-4-ones. Indian J Heterocycl Chem 15: 293-294. 
12. Hess HJ, Cronin TH, Scriabine (1968) A: Antihypertensive 2-amino4(3H)-quinazolinones. J Med Chem 11: 130-136.

13. Paneersalvam P, Raj T, Ishar PS, M Singh, B Sharma V (2010) Rather BA: Anticonvulsant activity of Schiff bases of 3-amino-6,8-dibromo-2phenylquinazolin-4(3H)-ones. Indian J Pharm Sci 72: 375-378.

14. J Chen, D Wu, F He, M Liu, H Wu, et al (2008) W. Su Tetrahedron Lett 49 3814-3818.

15. M Abdollahi-Alibeik, E Shabani, Chin (2011) Chem Lett 22: 1163-1166.

16. M Tajbakhsh, R Hosseinzadeh, P Rezaee, M Tajbakhsh, Chin (2014) J Catal 35: 58-65.

17. J Chen, D Wu, F He, M Liu, H Wu (2008) Tetrahedron Lett 49: 3814-3818.

18. A Bharathi, SM Roopan, A Kajbafvala, RD Padmaja, MS Darsana (2014) Chin Chem Lett 25: 324-326.

19. MT Maghsoodlou, N Khorshidi, MR Mousavi, N Hazeri, SM HabibiK. horassani (2014) Res Chem Intermed 41: 7497-7508.

20. Mahshid Hossaini, Reza Heydari, Malek Taher Maghsoodlou (2006) An efficient and convenient synthesis of quinazoline derivatives catalyzed by cyanuric chloride in water. Ir J of Cat 6(4): 363-368.

21. J Wu, X Du, J Ma, Y Zhang, Q Shi, et al. (2014) Green Chem 16: 3210-3217.
22. Wang, Shuliang Sheng, JieTu, Shujiang Wang, Xiangshan (2011) Chin J of Org Chem 31(9): 1522-1526.

23. DM Tal, D Keinan, Y Mazur, Tetrahedron (1981) 4327(b) A Onofrio A Scettri Synthesis (1985) 1159.

24. Y Kamitori, M Hojo, R Masuda, T Kimura, T Yoshida (1986) J Org Chem 5: 1427.

25. H Firouzabadi, N Iranpoor, H Hazarkhani, B Karimi (2002) J Org Chem 67: 2572.

26. KVNS Srinivas, I Mahender, B Das (2003) Synthesis 16: 2479.

27. M Sathe, AK Gupta, MP Kaushik (2006) Tetrahedron Lett 47: 3107.

28. HitendraKarade, ManishaSathe, MP Kaushik (2007) Catalysis Communications. 8: 741-746.

29. M Shioorkar, S Jadhav, M Ubale (2015) Simple Microwave assisted one pot synthesis of symmetrical curcumin analogues. Der Chemical Sinica 6(4): 110-113.

30.Zong-Bo Xie, Shi-Guo Zhang, Guo-Fang Jiang, Da-Zhao Sun, Zhang-Gao Le (2015) The green synthesis of 2,3-dihydroquinazolin-4(1H)-ones via direct cyclocondensation reaction under catalyst-free conditions. Green chem lett and review 8(3-4): 95-98.

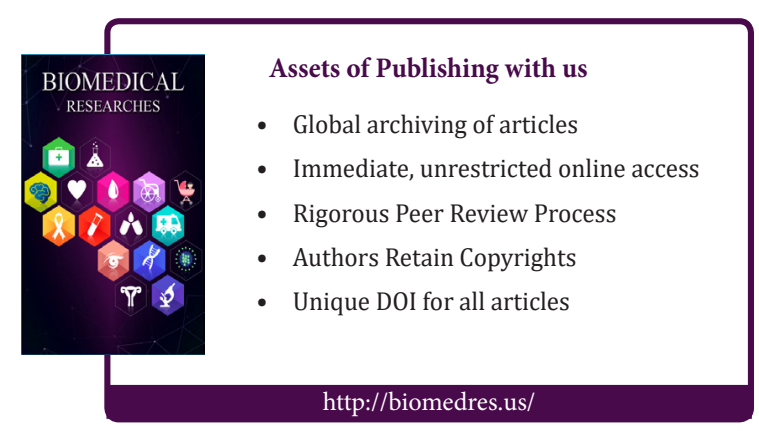

